\title{
Structural studies of human muscle FBPase*
}

\section{Jakub Barciszewski ${ }^{1}$, Kamil Szpotkowski ${ }^{1}$, Janusz Wiśniewski², Robert Kołodziejczyk², Dariusz Rakus², Mariusz Jaskolski ${ }^{1,3} \square$ and Andrzej Dzugaji,5®}

'Center for Biocrystallographic Research, Institute of Bioorganic Chemistry, Polish Academy of Sciences, Poznań, Poland; 2Department of Molecular Physiology and Neurobiology, Wroclaw University, Wrocław, Poland; '3 Department of Crystallography, Faculty of Chemistry, A. Mickiewicz University, Poznań, Poland; ${ }^{4}$ nnstitute of Genetics and Microbiology, Wroclaw University, Wrocław, Poland; ${ }^{5}$ Klodzko School of Medicine, Kłodzko, Poland

Muscle fructose-1,6-bisphosphatase (FBPase), which catalyzes the hydrolysis of fructose-1,6-bisphosphate (F1,6BP) to fructose-6-phosphate (F6P) and inorganic phosphate, regulates glucose homeostasis by controlling the glyconeogenic pathway. FBPase requires divalent cations, such as $\mathrm{Mg}^{2+}, \mathrm{Mn}^{2+}$, or $\mathrm{Zn}^{2+}$, for its catalytic activity; however, calcium ions inhibit the muscle isoform of FBPase by interrupting the movement of the catalytic loop. It has been shown that residue E69 in this loop plays a key role in the sensitivity of muscle FBPase towards calcium ions. The study presented here is based on five crystal structures of wild-type human muscle FBPase and its E69Q mutant in complexes with the substrate and product of the enzymatic reaction, namely F1,6BP and F6P. The ligands are bound in the active site of the studied proteins in the same manner and have excellent definition in the electron density maps. In all studied crystals, the homotetrameric enzyme assumes the same cruciform quaternary structure, with the $K$ angle, which describes the orientation of the upper dimer with respect to the lower dimer, of $-85^{\circ}$. This unusual quaternary arrangement of the subunits, characteristic of the R-state of muscle FBPase, is also observed in solution by small-angle X-ray scattering (SAXS).

Keywords: fructose-1,6-bisphosphatase; glycolysis; energy metabolism; active site; gluconeogenesis; glyconeogenesis; T/R-state enzyme;

Received: 27 November, 2020; revised: 23 December, 2020; accepted: 29 December, 2020 available on-line: 27 January, 2021

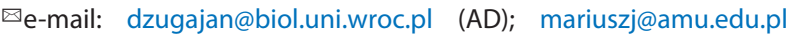
(MJ)

*Dedicated to Prof. Wladek Minor on the occasion of his 75th Birthday

Acknowledgements of Financial Support: This work was supported in part by the Polish National Science Centre (NCN) grant No. 2013/09/B/NZ1/01081. Research leading to these results has received funding from the European Community's Seventh Framework Programme (FP7/2007-2013) under BioStruct-X (grant agreement N 283570).

Abbreviations: FBPase, fructose-1,6-bisphosphatase; F1,6BP, fructose-1,6-bisphosphate; WT, wild type

\section{INTRODUCTION}

Fructose-1,6-bisphosphatase (FBPase, EC 3.1.3.11), which catalyzes the hydrolysis of fructose-1,6-bisphosphate $(\mathrm{F} 1,6 \mathrm{BP})$ to fructose-6-phosphate (F6P) and inorganic phosphate, is the main regulatory enzyme of gluconeogenesis and glyconeogenesis and thus it controls such fundamental processes as energy metabolism and glucose homeostasis (Tejwani, 1983; Gidh-Jain et al., 1994). Vertebrate genomes contain two distinct FBPase genes, FBP1 and FBP2, encoding two isozymes. Liver FBPase (or FBP1), the product of the FBP1 gene, is mainly expressed in the gluconeogenic organs, where it functions as a regulator of glucose synthesis from noncarbohydrates (Al-Robaiy \& Eschrich, 1999). Unlike liver FBPase, the muscle isoform (or FBP2), encoded by FBP2, is expressed in all vertebrate cells, whether glyconeogenic (e.g., in muscle fibers) or not (such as neurons, which reportedly do not synthesize glycogen from carbohydrate precursors) (Löffler et al., 2001).

Despite high level of similarity of their primary structure, the two isozymes play entirely different biological functions and significantly differ in such kinetic properties as susceptibility to inhibition (Dzugaj, 2006). FBPase activity is regulated by such physiological inhibitors as AMP and $\mathrm{NAD}^{+}$, which bind to an allosteric site, and fructose-2,6-bisphosphate (F2,6BP), which binds to the active site, as well as by calcium ions. The enzyme requires divalent cations such as $\mathrm{Mg}^{2+}$ or $\mathrm{Zn}^{2+}$ or $\mathrm{Mn}^{2+}$ for its activity (Gidh-Jain et al., 1994; Choe et al., 1998). Interestingly, calcium cations inhibit muscle FBPase, but not liver FBPase (Gizak et al., 2004; Zarzycki et al., 2007).

In line with its key role in glucose homeostasis, the activity of FBPase is under hormonal control (Bartrons et al., 1983; Pilkis et al., 1995). Glucagon and insulin, respectively, stimulate and inhibit the synthesis of F2,6BP, which acts as a competitive inhibitor of FBPase. F2,6BP acts synergistically with AMP to regulate the enzyme activity (Van Schaftingen \& Hers, 1981; Gidh-Jain et al., 1994; Choe et al., 1998). Muscle FBPase is about 100 times more susceptible to the action of the allosteric inhibitors AMP (Rakus et al., 2003) and NAD ${ }^{+}$(Rakus et al., 2003) than the liver isozyme, and about 1,000 times more sensitive to inhibition by $\mathrm{Ca}^{2+}$ (Gizak et al., 2004, 2008; Zarzycki et al., 2007). It was also shown that calcium ions inhibit muscle FBPase competitively in respect to $\mathrm{Mg}^{2+}$ and disrupt the Z-line-based FBPasealdolase complex in striated muscles, blocking glycogen re-synthesis during high-intensity exercise (Gizak et al., 2013). While the different sensitivity of muscle and liver FBPases to $\mathrm{Ca}^{2+}$ was shown to be dependent on the single E69Q point mutation (Zarzycki et al., 2007; Rakus et al., 2013), the structural differences leading to the unique responses to allosteric inhibitors and different ability to interact with various binding partners are not well understood. Recent studies of Wiśniewski and others (Wiśniewski et al., 2017) revealed that muscle FBPase exists in equilibrium between tetramers, dimers and monomers and that only the tetrameric form of FBPase is retained in the cell nucleus, whereas only the dimeric form 
associates with mitochondria and protects them against stress stimuli, such as elevated calcium and $\mathrm{H}_{2} \mathrm{O}_{2}$ levels.

Our knowledge about the structure of FBPase is predominantly based on studies of the enzyme isolated from porcine liver, while information about the muscle isoform is scarce and limited to only a few structural studies (Zarzycki et al., 2011; Shi et al., 2013, Barciszewski et al., 2016). Although Wiśniewski and collaborators demonstrated that in the absence of AMP muscle FBPase exists as a mixture of various oligomers (Wiśniewski et al., 2017), in all published crystal structures, the FBPases are homotetramers comprised of the upper (subunits $\mathrm{C} 1 \bullet \mathrm{C} 2$ ) and lower $(\mathrm{C} 3 \bullet \mathrm{C} 4)$ dimers, which rotate with respect to each other during the catalytic cycle. Our earlier studies revealed that the structure of the inactive (T-state), AMP-saturated muscle FBPase is practically identical to that of the T-state liver isozyme (Zarzycki et al., 2011; Barciszewski et al., 2016; Ruf et al., 2016), with the two dimers forming a small angle $x$. Based on the previously reported structures (known at that time for the liver isoform only) of the active R-state, we suggested that the explanation of the markedly different kinetic properties of the liver and muscle isoforms must reside in different quaternary arrangements of the active R-states of the isozymes. The crystal structure of the R-state of the muscle isozyme revealed (Barciszewski et al., 2016) that its cross-like form is indeed diametrically different from the flat form of the R-state of the liver isozyme. The cruciform R-state of muscle FBPase is stabilized by a hydrophobic motif, termed the "leucine lock", which plays an important role in the R-to- $\mathrm{T}$ transition, and in particular blocks the residue (D187) responsible for parking the catalytic loop in the disengaged (inactive) conformation when the enzyme is in the T-state (Barciszewski et al., 2016). The leucine lock is not present in the liver isozyme. All these observations have led us to several important questions: is the perpendicular R-state also present in solution? What is the R-state structure of the E69Q mutant? Are there any differences in substrate/ product binding between the wild-type and E69Q muscle FBPase?

To address these questions, here we resolved and present the first crystal structures of both the wild-type and E69Q muscle FBPase in its active R-state in complexes with F1,6BP and F6P, which are, respectively, the substrate and the product of the enzyme. The results show that the arrangement of the substrate/product binding site in the two proteins is identical and that the sensitivity to calcium ions is not related to direct disruption of the F1,6BP/F6P binding site. Our SAXS experiments confirm that in solution muscle FBPase forms the crosslike quaternary arrangement known from crystallographic studies, thus supporting the conclusion about physiologically different R-states of the two isoforms.

\section{MATERIALS AND METHODS}

\section{Protein expression, purification and crystallization}

Mutagenesis, expression and purification of wild type (W'T) human muscle FBPase and its E69Q mutant were carried out as described previously (Rakus et al., 2003; Zarzycki et al., 2007). AMP was removed from protein samples by extensive dialysis and FPLC gel filtration on a HiLoad Superdex 200 16/60 column (GE Healthcare), as described previously (Barciszewski et al., 2016).

Crystallization experiments were carried out at $292 \mathrm{~K}$ using the hanging-drop vapor-diffusion method. Protein and precipitant solutions were mixed at 1:1 volume ratio to form $3 \mu \mathrm{l}$ drops. Samples of catalytically active WT FBPase and its E69Q mutant without any ligands were concentrated to $6 \mathrm{mg} \mathrm{ml}^{-1}$ and crystallized using $10 \mathrm{mM}$ Tris buffer $\mathrm{pH} 7.4$, containing $10 \mathrm{mM} \mathrm{MgCl}_{2}, 2 \mathrm{M} \mathrm{NaCl}$ and $10 \%(\mathrm{v} / \mathrm{v})$ PEG6000. The best crystals grew to the dimensions of $0.20 \times 0.15 \times 0.15 \mathrm{~mm}$ within three months. Two soaking experiments were performed using crystals of both WT and E69Q FBPase. In the soaking experiment the mother liquor was supplemented with $5 \mathrm{mM}$ fructose-6-phosphate (F6P) or $10 \mathrm{mM}$ fructose-1,6-bisphosphate (F1,6BP), with soaking times of 2.5 h. Each soaking solution was additionally supplemented with $100 \mathrm{mM} \mathrm{CaCl}_{2}$ to inhibit the enzyme activity.

\section{$\mathrm{X}$-ray data collection and processing}

For data collection, the crystals were cryoprotected by immersion for a few seconds in the mother liquor supplemented with $20 \%$ (v/v) glycerol and, where appropriate, with the soaking ligands, and then flash-vitrified at $100 \mathrm{~K}$ in a cold nitrogen-gas stream. X-Ray diffraction data were collected for five crystals (maximum resolution in parentheses): (i) WT with F6P (1.92 $\AA$ ), (ii) WT with F1,6BP (2.19 $\AA$ ), (iii) E69Q without any ligand

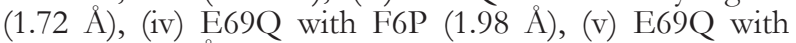
F1,6BP (2.30 $\AA$ ). In all cases, synchrotron radiation was used, provided by MX beamlines of the BESSY II (Berlin, Germany) synchrotron, equipped with Rayonix MX225 (square) or Ryonix SX-165 (round) CCD detectors. The diffraction data for E69Q in complex with F1,6BP were processed and scaled with the HKL-2000 package (Otwinowski \& Minor, 1997) while XDSAPP (Kabsch, 2010; Krug et al., 2012) was used in all other cases. The data collection statistics are presented in Table 1.

\section{Structure determination and refinement}

Both, the WT and mutant E69Q muscle FBPase crystallized in the same space group $I 4_{1} 22$, isomorphously with the wild-type human muscle FBPase (PDB: 5ET5) described previously (Barciszewski et al., 2016), with one protein molecule in the asymmetric unit, located near the crystallographic 222 site. In several rounds of manual rebuilding in COOT (Emsley et al., 2010), starting with the coordinates of $5 \mathrm{ET} 5$, the models were corrected according to the electron density maps, with special emphasis on the N-terminal fragment of the protein molecules. The refinement of all structures was carried out in phenix.refine (Afonine et al., 2012). Riding hydrogen atoms of the protein molecules were included in $F_{c}$ calculations for all structures. TLS parameters (Winn et al., 2001; Painter \& Merritt., 2006) were refined for four (WT/ F6P), seven (WT/F1,6BP), three (E69Q), two (E69Q/ F6P) and three (E69Q/F1,6BP) rigid groups per subunit, as suggested by the refinement program. Water molecules $(94,25,59,61$ and 12, respectively) were added to the models after manual validation of their electron density and hydrogen bonding. The refinement statistics are presented in Table 1.

\section{PDB accession codes and raw data deposition}

Atomic coordinates (.pdb) and processed structure factors (.mtz) corresponding to the final models presented in this work have been deposited with the Protein Data Bank (PDB) under the accession codes 5ET8 (R-state muscle FBPase in complex with F6P); 5K56 (R-state muscle FBPase in complex with F1,6BP); 5K55 (R-state E69Q mutant FBPase in complex with F6P); 
Table 1. Data collection and refinement statistics.

\begin{tabular}{|c|c|c|c|c|c|}
\hline Crystal & WT/F6P & WT/F1,6BP & $\mathrm{E} 69 \mathrm{Q}$ & E69Q/F6P & $\mathrm{E} 69 \mathrm{Q} / \mathrm{F} 1,6 \mathrm{BP}$ \\
\hline \multicolumn{6}{|l|}{ Data collection } \\
\hline Radiation source & BESSY II, Berlin & & & & \\
\hline Beamline & 14.1 & 14.3 & 14.1 & 14.1 & 14.3 \\
\hline Wavelength $(\AA)$ & 0.918 & 0.894 & 0.918 & 0.918 & 0.894 \\
\hline Temperature (K) & 100 & 100 & 100 & 100 & 100 \\
\hline Space group & 14,22 & 14,22 & 14,22 & 14,22 & 14,22 \\
\hline Unit cell parameters $(\AA ̊)$ & $\begin{array}{l}a=b=72.11 \\
c=232.00\end{array}$ & $\begin{array}{l}a=b=72.58 \\
c=232.44\end{array}$ & $\begin{array}{l}a=b=72.15 \\
c=235.06\end{array}$ & $\begin{array}{l}a=b=72.15 \\
c=235.06\end{array}$ & $\begin{array}{l}a=b=72.45 \\
c=233.95\end{array}$ \\
\hline Resolution $(\AA)$ & $\begin{array}{l}46.68-1.92 \\
(2.04-1.92)^{a}\end{array}$ & $\begin{array}{l}34.64-2.19 \\
(2.33-2.19)\end{array}$ & $\begin{array}{l}34.48-1.72 \\
(1.82-1.72)\end{array}$ & $\begin{array}{l}32.03-1.98 \\
(2.10-1.98)\end{array}$ & $\begin{array}{l}31.03-2.30 \\
(2.38-2.30)\end{array}$ \\
\hline Reflections collected/unique & $229018 / 23929$ & $155047 / 16183$ & $308771 / 33575$ & $210365 / 22004$ & $210365 / 13945$ \\
\hline Completeness (\%) & $99.8(99.4)$ & $98.8(95.4)$ & $99.5(97.1)$ & $99.9(99.5)$ & $93.9(69.5)$ \\
\hline Multiplicity & $9.57(9.76)$ & $9.58(9.48)$ & $9.20(7.00)$ & $9.56(9.61)$ & $8.7(5.7)$ \\
\hline$R_{\text {merge }}^{\mathrm{b}}$ & $0.076(0.896)$ & $0.066(0.748)$ & $0.055(0.793)$ & $0.076(0.761)$ & $0.076(0.622)$ \\
\hline$<I / \sigma(I)>$ & $19.05(2.57)$ & $28.03(3.55)$ & $23.67(2.25)$ & $22.79(3.15)$ & $22.79(3.15)$ \\
\hline \multicolumn{6}{|l|}{ Refinement } \\
\hline Unique reflections, work/test & $22900 / 1028$ & $15140 / 1043$ & $32575 / 1000$ & $21004 / 1000$ & $13014 / 931$ \\
\hline Matthews volume $\left(\AA^{3} \mathrm{Da}^{-1}\right) /$ solvent $(\%)$ & $2.06 / 40.3$ & $2.4 / 48.9$ & $2.09 / 41.1$ & $2.06 / 40.4$ & $2.31 / 46.9$ \\
\hline $\begin{array}{l}\text { No. of non-H atoms, protein/ligand/ } \\
\text { solvent }\end{array}$ & $2225 / 16 / 94$ & $2108 / 20 / 25$ & $2165 / 0 / 59$ & $2223 / 16 / 61$ & $2142 / 20 / 12$ \\
\hline$R_{\text {work }} / R_{\text {free }}(\%)$ & $17.96 / 22.33$ & $20.50 / 27.98$ & $21.38 / 24.90$ & $18.53 / 23.49$ & $21.50 / 28.80$ \\
\hline \multicolumn{6}{|l|}{ RMSD from ideal geometry } \\
\hline bond lengths $(\AA)$ & 0.018 & 0.009 & 0.019 & 0.017 & 0.009 \\
\hline bond angles (०) & 1.71 & 1.14 & 1.68 & 1.62 & 1.22 \\
\hline \multicolumn{6}{|l|}{ Ramachandran statistics (\%) } \\
\hline favored & 98.2 & 97.7 & 97.1 & 97.5 & 96.65 \\
\hline allowed & 1.8 & 2.3 & 2.9 & 2.5 & 2.97 \\
\hline PDB code & 5ET8 & $5 K 56$ & $5 K 54$ & $5 K 55$ & $5 \mathrm{LOA}$ \\
\hline
\end{tabular}

aValues in parentheses correspond to the highest resolution shell.

${ }^{\mathrm{b}} R_{\text {merge }}=\sum_{h k l} \sum_{i} \mid I_{i}(h k l)-\langle I(h k l)| / \sum_{h k l} \sum_{i} I_{i}(h k l)$, where $I_{i}(h k l)$ is the intensity of observation $i$ of reflection $h k l$.

5L0A (R-state E69Q mutant FBPase in complex with F1,6BP); and 5K54 (R-state E69Q mutant FBPase without any ligands). The corresponding raw X-ray diffraction images have been deposited in the RepOD Repository at the Interdisciplinary Centre for Mathematical and Computational Modelling (ICM) of the University of Warsaw, Poland, and are available for download with the following Digital Object Identifiers (DOI): http:/ / dx.doi.org/10.18150/2073150 (R-state muscle FBPase in complex with F6P); http://dx.doi.org/10.18150/7369446 (R-state muscle FBPase in complex with F1,6BP); http://dx.doi.org/10.18150/2931163 (R-state E69Q mutant FBPase in complex with F6P); http://dx.doi. org/10.18150/3968588 (R-state E69Q mutant FBPase with F1,6BP); and http://dx.doi.org/10.18150/3279048 (R-state E69Q mutant FBPase without any ligands).

\section{SAXS experiments in solution}

Wild-type FBPase for the experiments in solution was prepared in the same way as the sample for crystallization experiments described previously (Barciszewski et al., 2016). The SAXS measurements were performed at three different concentrations $(1 \mathrm{mg} / \mathrm{ml} ; 4 \mathrm{mg} / \mathrm{ml} ; 8 \mathrm{mg} / \mathrm{ml})$ using $25 \mathrm{mM}$ Hepes buffer $\mathrm{pH}$ 7.0. Small-angle X-ray scattering data were collected on the bio-SAXS beamlines P12 (Petra-III, EMBL/DESY, Hamburg, Germany) and I911-4 (MAX-Lab, Lund, Sweden). Samples of $20 \mu \mathrm{l}$ protein solution and of the corresponding matching buffer were analyzed. SAXS data were collected at $300 \mathrm{~K}$ over the $q$ range of $0.00088-0.5 \AA^{-1}$ (DESY) or 0.01-0.45 $\AA^{-1}$ (MAX-Lab), and overlays of the merged data sets were used to detect concentration-dependent scattering in the lowest $q$ region. All SAXS data were processed using the ATSAS package (Petoukhov et al., 2012). The CRYSOL program (Svergun et al., 1995) was applied for the evaluation of the solution scattering patterns using the crystallographic FBPase models. Ab initio modelling was performed with $D A M M I N$ (Svergun et al., 1999). The SAXS data collection and structural parameters are presented in Table 2.

\section{RESULTS}

\section{Overall structure}

All five structures of the WT and E69Q mutant protein presented in this report were solved in space 
Table 2. SAXS data collection and structural parameters obtained from SAXS scattering derived parameters.

\begin{tabular}{|c|c|c|c|c|}
\hline FBPase state & $\mathrm{R}$ & $\mathrm{T}$ & $\mathrm{R}$ & $\mathrm{T}$ \\
\hline \multicolumn{5}{|l|}{ Data collection parameters } \\
\hline Instrument & P12 Petra-III & & \multicolumn{2}{|c|}{ 1911-4 MAX-Lab } \\
\hline Wavelength $(\AA ̊)$ & 1.24 & & \multicolumn{2}{|l|}{0.91} \\
\hline$q$ range $\left(\AA^{-1}\right)$ & $0.00088-0.5$ & & \multicolumn{2}{|l|}{$0.01-0.45$} \\
\hline Exposure time (s) & 1 & & \multicolumn{2}{|l|}{120} \\
\hline Concentration range $(\mathrm{mg} / \mathrm{ml})$ & $1-8$ & & \multicolumn{2}{|l|}{$1-8$} \\
\hline Temperature (K) & 293 & & \multicolumn{2}{|l|}{293} \\
\hline \multicolumn{5}{|l|}{ Structural parameters } \\
\hline $\mathrm{I}(0)$ (arbitrary units) [from $\mathrm{P}(\mathrm{r})]$ & $46.35 \pm 1$ & $46.37 \pm 1$ & $163.7 \pm 1.7$ & $163.2 \pm 1.7$ \\
\hline$R_{g}(\AA)[$ from $P(r)]$ & 35.9 & 34.3 & 35.9 & 34.3 \\
\hline $\mathrm{R}_{\mathrm{g}}(\AA)$ (from Guinier) & 35.9 & 34.3 & 35.9 & 34.3 \\
\hline$D_{\max }(\AA)$ & 101 & 115 & 101 & 115 \\
\hline Porod volume estimate $\left(\AA^{3}\right)$ & 180015 & 180201 & 180120 & 180191 \\
\hline Dry volume calculated from sequence $(\AA 3)$ & 179516 & 179516 & 179516 & 179516 \\
\hline \multicolumn{5}{|l|}{ Molecular mass determination } \\
\hline Contrast $\left(\Delta \rho \times 10^{10} \mathrm{~cm}^{-2}\right)$ & \multicolumn{4}{|l|}{3.047} \\
\hline Molecular mass $\mathrm{M}_{\mathrm{r}}$ [from I(0)] (kDa) & \multicolumn{4}{|l|}{148} \\
\hline Monomeric $\mathrm{M}_{\mathrm{r}}$ calculated from sequence $(\mathrm{kDa})$ & \multicolumn{4}{|l|}{36.842} \\
\hline \multicolumn{5}{|l|}{ Software used } \\
\hline Primary data reduction & \multicolumn{4}{|l|}{ PRIMUS } \\
\hline Data processing & \multicolumn{4}{|l|}{ PRIMUS } \\
\hline$A b$ initio analysis & \multicolumn{4}{|l|}{ DAMMIN } \\
\hline Validation and averaging & \multicolumn{4}{|l|}{ DAMAVER } \\
\hline Computation of model intensities & \multicolumn{4}{|l|}{ CRYSOL } \\
\hline 3D graphics representation & \multicolumn{4}{|l|}{ PyMOL } \\
\hline
\end{tabular}

group $I 4_{1} 22$. They are isomorphous with the structure of muscle FBPase in the R-state (PDB: 5ET5) described previously (Barciszewski et al., 2016). In each crystal, there is one protein subunit in the asymmetric unit and the 222-symmetric homotetramer is generated by crystallographic symmetry. The overall structures are similar to that described previously (Barciszewski et al., 2016), as illustrated by the r.m.s.d. of their $C \alpha$ superpositions $(0.15-0.18 \AA)$ when compared with the reference R-state structure 5ET5 of human muscle FBPase. The topology of the $\alpha$-helices and the $\beta$-sheet is also conserved. Several amino acid residues (including the E69Q mutation) could not be traced in the electron density maps. In particular, the catalytic loop L2 (residues 50-73) is in the disordered state in all the structures presented here.

\section{Tetramer architecture}

Superpositions of all five WT and E69Q mutant FBPase tetramers from this study onto the reference human muscle R-state tetramer show no change in the te- tramer conformation. The upper $\mathrm{C} 1 \bullet \mathrm{C} 2$ dimer is rotated by $x=-85^{\circ}$ relative to the lower $\mathrm{C} 3 \bullet \mathrm{C} 4$ dimer in all five structures presented here.

\section{The active site}

Among the presented structures, there are two types of active-site complexes, with the catalytic reaction substrate $(\mathrm{F} 1,6 \mathrm{BP})$ or product $(\mathrm{F} 6 \mathrm{P})$ at full occupancy. The excellent electron density leaves no doubt that all of these ligand molecules are the $\beta$ anomers of the fructose furanose ring (Fig. 1). Both ligand molecules are stabilized in the active site by a network of direct hydrogen bonds formed by residues Asn212, Tyr215, Tyr244, Met248, Tyr264 and Lys274, as listed in Table 3. Additionally, Asp251 forms a water-mediated hydrogen bond with the fructose hydroxyl O4 atom (Fig. 2). Each ligand molecule is also hydrogenbonded with the side chain of Arg243 from the complementary subunit within the tight dimer (upper or lower). 
A

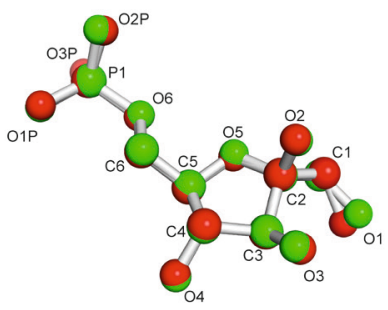

C

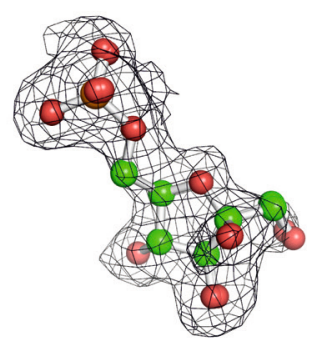

E

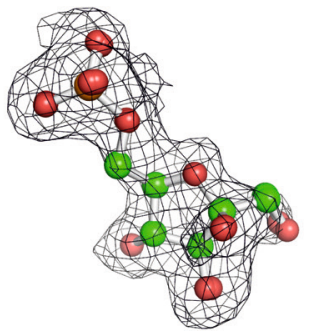

B

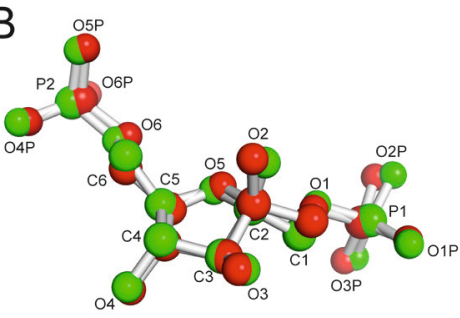

D

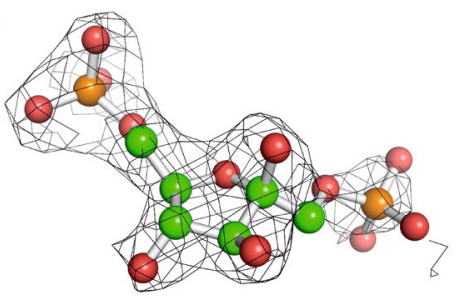

$\mathrm{F}$

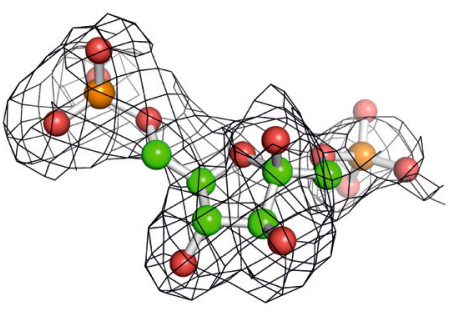

Figure 1. Superposition of fructose-6-phosphate (A) and fructose-1,6-bisphosphate (B) molecules from their complexes with WT (green) and $\mathrm{E} 69 \mathrm{Q}$ mutant (red) muscle $\mathrm{FBPase}$.

The ligand molecules F6P $(\mathbf{C}, \mathbf{E})$ and $\mathrm{F} 1,6 \mathrm{BP}(\mathbf{D}, \mathbf{F})$ are shown in their $F_{0}-F_{c}$ OMIT electron density maps for complexes with WT FBPase (left panels) and E69Q FBPase (right panels). The electron density maps are contoured at the $2 \sigma(\mathbf{C}), 3 \sigma(\mathbf{D}), 2 \sigma(\mathbf{E})$ and $1.5 \sigma(\mathbf{F})$ level.

\section{Quaternary structure in solution}

The experimental SAXS curves collected for the FBPase sample in $25 \mathrm{mM}$ Hepes buffer $\mathrm{pH} 7.0$ are shown in Fig. 3. The SAXS analysis and structural parameters confirmed the existence of the $\mathrm{R}$ and $\mathrm{T}$ states in solution. Low-resolution SAXS models of both states superimposed with the crystal structures are shown in Fig. 4. The conformational transformation from the $\mathrm{T}$ to $\mathrm{R}$ state is induced by removal of AMP, in agreement with the previous crystallographic study (Barciszewski et al., 2016).

\section{DISCUSSION}

Despite a number of crystallographic and biochemical studies, the structure of FBPase in solution has not been investigated before. The studies of Wisniewski and others (Wisniewski et al., 2017), which used analytical centrifugation, demonstrated that muscle FBPase exists in equilibrium of tetramers, dimers and monomers, when the allosteric inhibitor AMP is absent. Addition of AMP induces tetramerization of practically the whole population of the enzyme molecules (Wiśniewski et al., 2017). Our SAXS studies unambiguously show that muscle FBPase exists in solution in both, the $\mathrm{T}$ and $\mathrm{R}$ states that are identical as in the crystallized enzyme (Barciszewski et al., 2016).
The crystal structures of human muscle W'T and E69Q FBPase in the $\mathrm{R}$ state presented here show that upon removal of AMP from the crystallization solution, the enzyme assumes the cruciform conformation regardless of the presence or absence of the substrate or the product of the catalytic reaction.

The binding mode of the ligands (F6P, F1,6BP) in the active site of the muscle isozyme closely resembles the situation known for the liver isozyme. Additionally, we have analyzed the structure of the mammalian enzymes available in the PDB and found that the residues involved in substrate/product binding are conserved among all vertebrate FBPases. Specifically, Asn212, Asn215, Arg243, Tyr244, Leu248, Tyr264 and Lys274 (human muscle FBPase residue numbers) form a hydrogen bond network with the C6 phosphate group and the furanose ring of the ligand molecules in the active site (Fig. 2). Residues involved in the catalytic metal $\left(\mathrm{Mg}^{2+}\right)$ coordination (Glu68, Glu97, Glu98, Asp118, Asp121, Glu280) are also conserved in vertebrate FBPases. Together with the phosphate group at the $\mathrm{C} 1$ atom of the F1,6BP substrate molecule, these residues form a huge acid cluster which is essential for divalent cation recognition. However, despite their presence in the crystallization solution, no $\mathrm{Mg}^{2+}$ ions could be detected in the presented crystal structures. This might have been presumably caused by the presence of calcium ions in the soaking solutions, which impede transition of loop 
Table 3. Hydrogen bonds ( $\AA$ ) between residues of WT and E69Q FBPase and fructose-1,6-bisphosphate and fructose-6-phosphate

\begin{tabular}{|c|c|c|c|c|c|}
\hline \multirow[t]{2}{*}{ Molecule } & \multirow[t]{2}{*}{ Atom } & \multicolumn{2}{|c|}{ Distance $[\AA ̊]$} & \multirow[t]{2}{*}{ Atom } & \multirow[t]{2}{*}{ Residue } \\
\hline & & WT & E69Q & & \\
\hline \multirow{11}{*}{$\mathrm{F} 1,6 \mathrm{BP}$} & $\mathrm{O} 4 \mathrm{P}(\mathrm{P} 2)$ & 2.74 & 2.40 & $\mathrm{Nn} 2$ & $\operatorname{Arg} 243(C 2)$ \\
\hline & O6P(P2) & 2.90 & 2.80 & $\mathrm{~N} \gamma 2$ & Asn212 \\
\hline & 01 & 3.51 & 3.82 & $\mathrm{~N} \varepsilon$ & Lys274 \\
\hline & $\mathrm{O} 5$ & 2.77 & 2.83 & $\mathrm{~N} \varepsilon$ & Lys274 \\
\hline & $\mathrm{O} 6$ & 3.16 & 3.66 & $\mathrm{~N} \varepsilon$ & Lys274 \\
\hline & $\mathrm{O} 3$ & 2.60 & 2.64 & $\mathrm{~N}$ & Met248 \\
\hline & $\mathrm{O} 4$ & 3.21 & 3.43 & $\mathrm{~N}$ & Met248 \\
\hline & O5P(P2) & 2.49 & 2.86 & $\mathrm{OH}$ & Tyr215 \\
\hline & O6P(P2) & 2.74 & 2.58 & $\mathrm{OH}$ & Tyr244 \\
\hline & O5P(P2) & 2.63 & 2.68 & $\mathrm{OH}$ & Tyr264 \\
\hline & $\mathrm{O} 4$ & 2.52 & 2.72 & $\mathrm{O}$ & Wat \\
\hline \multirow[t]{2}{*}{ Wat } & $\mathrm{O}$ & 2.68 & 2.72 & $0 \gamma 2$ & Asp251 \\
\hline & & WT & E69Q & & \\
\hline \multirow{10}{*}{ F6P } & $\mathrm{O} 4 \mathrm{P}(\mathrm{P} 2)$ & 2.71 & 2.80 & $\mathrm{Nn} 2$ & $\operatorname{Arg} 243(C 2)$ \\
\hline & O6P(P2) & 2.94 & 2.90 & $\mathrm{~N} \gamma 2$ & Asn212 \\
\hline & $\mathrm{O} 6$ & 2.89 & 3.04 & $\mathrm{~N} \varepsilon$ & Lys274 \\
\hline & $\mathrm{O} 5$ & 2.76 & 2.96 & $\mathrm{~N} \varepsilon$ & Lys274 \\
\hline & $\mathrm{O} 3$ & 2.80 & 2.83 & $\mathrm{~N}$ & Met248 \\
\hline & $\mathrm{O} 4$ & 3.27 & 3.25 & $\mathrm{~N}$ & Met248 \\
\hline & O5P(P2) & 2.48 & 2.58 & $\mathrm{OH}$ & Tyr215 \\
\hline & O6P(P2) & 2.61 & 2.68 & $\mathrm{OH}$ & Tyr244 \\
\hline & O5P(P2) & 2.56 & 2.58 & $\mathrm{OH}$ & Tyr264 \\
\hline & $\mathrm{O} 4$ & 2.70 & 2.59 & $\mathrm{O}$ & Wat \\
\hline Wat & $\mathrm{O}$ & 2.62 & 2.72 & Oү2 & Asp251 \\
\hline
\end{tabular}

L2 (residues 50-72) into the engaged state, in which it would take part in the formation of the acid cluster.

An intriguing, albeit still open, question is which fructose anomer is the natural substrate of FBPase when the catalytic reaction actually takes place. In solution, fructose-1,6-bisphosphate, like other similar carbohydrates, exists in an equilibrium of the $\alpha$-anomer $(15 \%), \beta$-anomer $(81 \%)$ and small amounts of some other forms (4\%) (Frey et al., 1977). Based on such equilibrium binding studies, it was shown that an analogue of $\beta$-D-fructose-1,6bisphosphate binds to the enzyme ten times more tightly than its $\alpha$-anomer (Marcus, 1976). However, it was also shown that high concentration of $\beta$-fructose-6-phosphate inhibits the activity of FBPase (Benkovic \& deMaine, 1982; Villeret et al., 1995). The studies of Benkovic and colleagues, also revealed that liver FBPase may utilize the $\beta$-anomer of F1,6BP as substrate, however, at a rate that was 5- to 10-times lower than what was measured for the $\alpha$-anomer (Benkovic et al., 1974). The latter finding might suggest that the $\alpha$-anomer is the preferred physiological substrate of FBPase, and this hypothesis seemed to be supported by the crystal structure of the E69Q mu- tant of human muscle FBPase in complex with AMP and F6P, which was interpreted with the $\alpha$-anomer of the F6P product in the active site (PDB deposit 3IFC) (Kolodziejczyk et al., 2011). However, a re-refinement of that 3IFC model indicates that the F6P ligand molecule can fit the 1.9 $\AA$ electron density maps equally well in the $\alpha$ and $\beta$ configurations (Barciszewski et al., unpublished results) (Fig. 5).

On the other hand, experiments on complex formation between muscle FBPase and muscle aldolase apparently supported the hypothesis that only the $\alpha$-anomer is the physiological substrate of FBPase. These experiments demonstrated that only aldolase-associated FBPase will catalyze the reaction of $\mathrm{F} 1,6 \mathrm{BP}$ hydrolysis in the presence of physiological concentration of the AMP inhibitor (Rakus et al., 2003), and that the aldolase may directly cascade the newly synthesized fructose-1,6-bisphosphate down to the FBPase in a process called substrate channeling (Rakus et al., 2004). Since it has been conclusively shown that aldolase synthesizes the $\alpha$-anomer of F1,6BP (Penhoet et al., 1969) it might be speculated that the $\alpha$-anomer is the main physiological substrate of FBPase. 


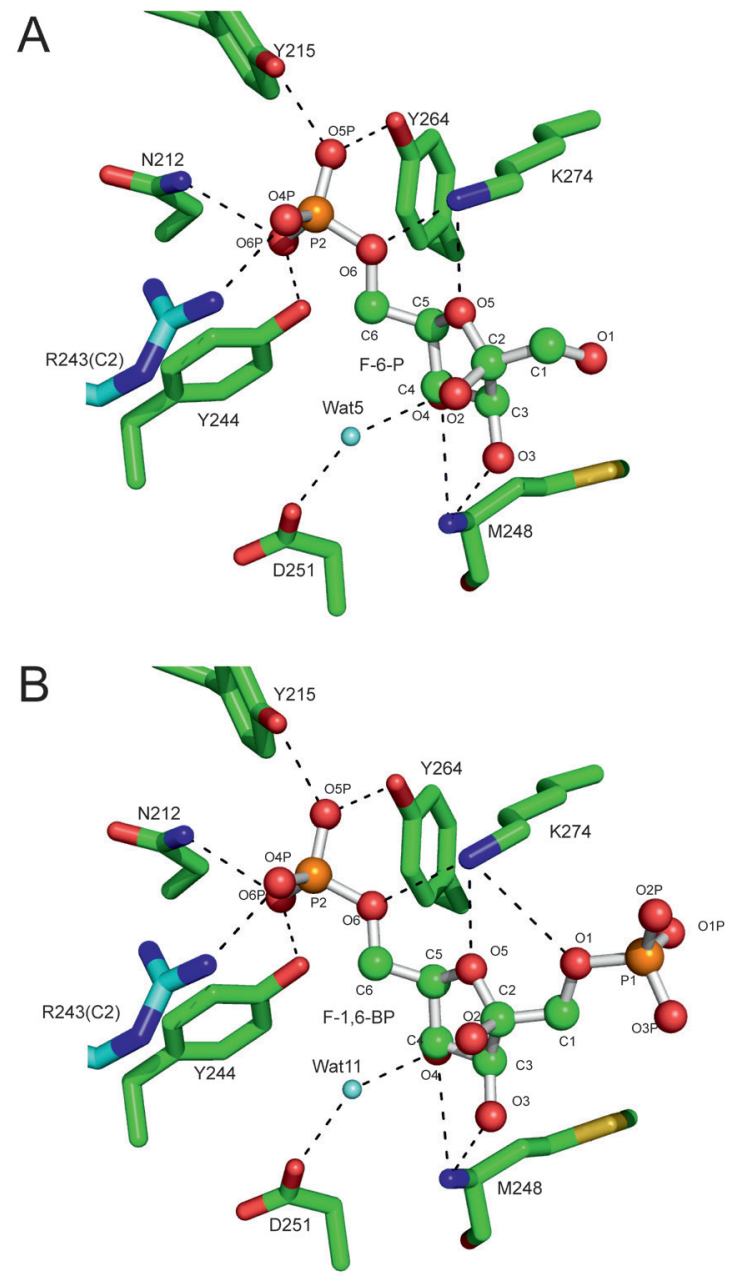

Figure 2. Comparison of the F6P (A) and F1,6BP (B) binding modes in WT FBPase.

The ligand binding modes in the $\mathrm{E} 69 \mathrm{Q}$ mutant protein are identical with those illustrated here. Residues from subunits $C 1 / C 2$ are colored green/blue, main-chain atoms have been omitted for clarity (except for M248).
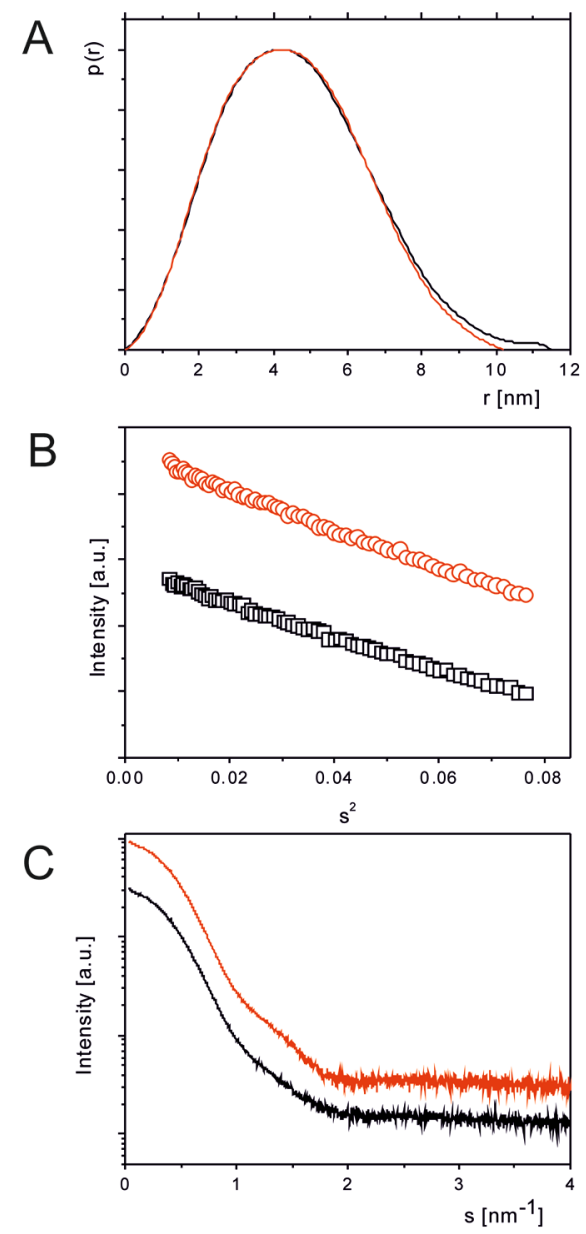

Figure 3. Experimental SAXS curves (A), Guinier plots (B), and pair distribution functions (C) for R- (red) and T-state (black) muscle FBPase.

\section{A}

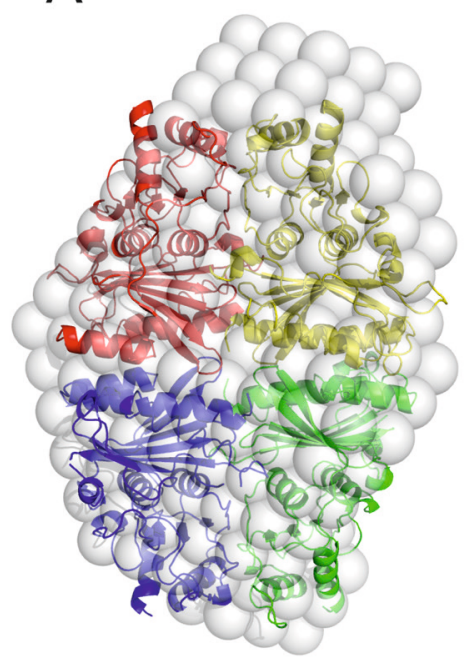

B

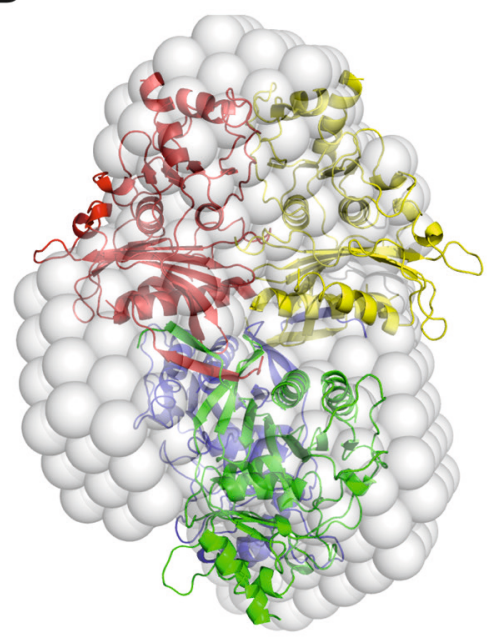

Figure 4. Comparison of the crystal structures (cartoon model) and low-resolution SAXS structures (semitransparent surface) of muscle FBPase in the T-state (A) and R-state (B).

The crystal structures of the T-state (5ET6) and R-state (5ET5) were presented previously (Barciszewski et al., 2016). 
A

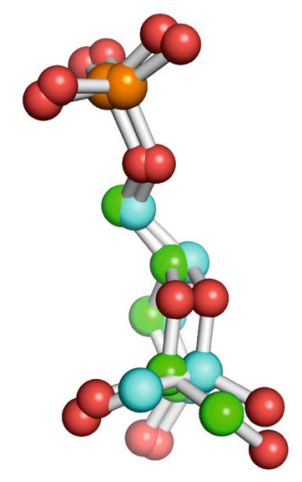

B

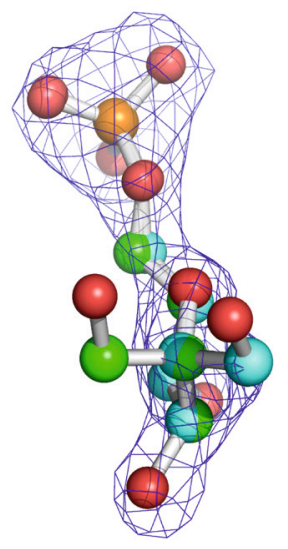

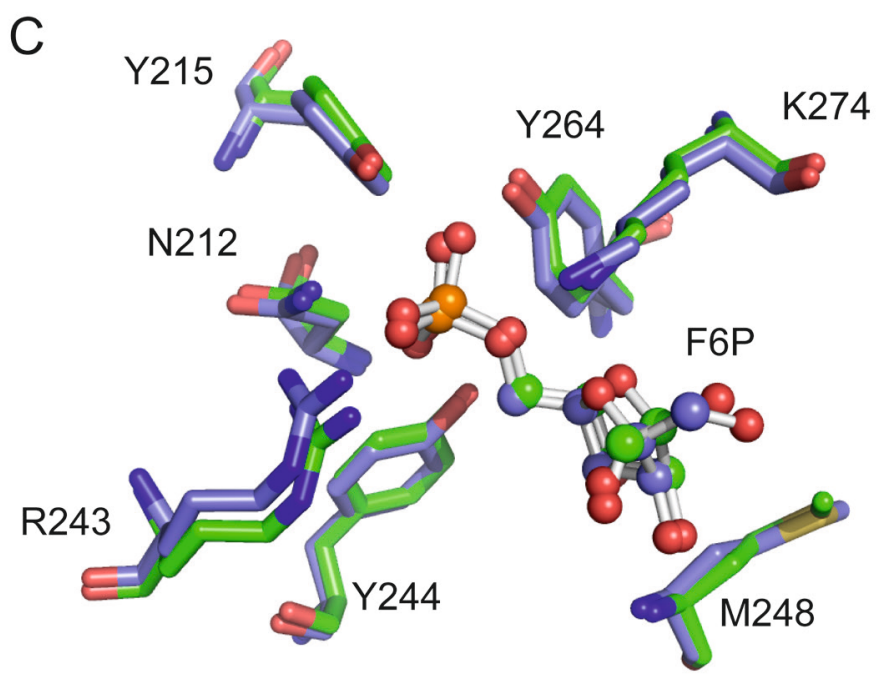

Figure 5. (A) Superposition of $a$-fructose-6-phosphate (green) and $\beta$-fructose-6-phosphate (blue) from their complexes with E690 (3IFC) and WT human muscle FBPase (5ET8), respectively. (B) Superposition of the originally modeled (3IFC) a-fructose-6-phosphate (green) and reinterpreted $\beta$-fructose-6-phosphate (blue) from the complex with E69Q FBPase.

The $F_{0}-F_{c}$ OMIT electron-density map is contoured at the $2 \sigma$ level. (C) Superposition of FBPase amino acid residues involved in ligand binding of a-fructose-6-phosphate (3IFC, green) and $\beta$-fructose-6-phosphate (WT protein, this work, blue). The hydrogen bond lengths are listed in Table 4.

Table 4. Comparison of hydrogen bonds ( $\AA$ ) between residues of WT (this work) and E69Q mutant (PDB ID 3IFC) FBPase C1 subunit and anomers of fructose-6-phosphate.

Additional contacts with subunit C2 are in italics.

\begin{tabular}{|c|c|c|c|c|c|}
\hline \multicolumn{3}{|c|}{ E69Q FBPase - a-F6P (3IFC) } & \multicolumn{3}{|c|}{ WT FBPase $-\beta-F 6 P$} \\
\hline P6P[O3] & 2.81 & Met248[N] & Met248[N] & 2.78 & $\mathrm{~F} 6 \mathrm{P}[\mathrm{O} 3]$ \\
\hline $\mathrm{P} 6 \mathrm{P}[05]$ & 2.67 & Lys $274[N \varepsilon]$ & Lys $274[N \varepsilon]$ & 2.78 & $\mathrm{~F} 6 \mathrm{P}[05]$ \\
\hline P6P[06] & 2.90 & Lys $274[N \varepsilon]$ & Lys $274[N \varepsilon]$ & 2.90 & F6P[06] \\
\hline P6P[O3P] & 2.88 & Asn212 [Ny2] & Asn212 [Nץ2] & 2.96 & $\mathrm{~F} 6 \mathrm{P}[\mathrm{O} 1 \mathrm{P}]$ \\
\hline P6P[O3P] & 2.71 & Tyr244[OH] & Tyr244[OH] & 2.63 & $\mathrm{~F} 6 \mathrm{P}[\mathrm{O} 1 \mathrm{P}]$ \\
\hline P6P[01P] & 2.74 & Tyr264[OH] & Tyr264[OH] & 2.59 & $\mathrm{~F} 6 \mathrm{P}[\mathrm{O} 2 \mathrm{P}]$ \\
\hline P6P[01P] & 2.65 & Tyr215[OH] & Tyr215[OH] & 2.46 & $\mathrm{~F} 6 \mathrm{P}[\mathrm{O} 2 \mathrm{P}]$ \\
\hline P6Р[О3Р] & 3.65 & $\operatorname{Arg} 243[N \eta 1]$ & $\operatorname{Arg} 243[N \eta 1]$ & 3.68 & F6P[O1P] \\
\hline$P 6 P[O 2 P]$ & 2.68 & $\operatorname{Arg} 243[\mathrm{Nn} 2]$ & $\operatorname{Arg} 243[\mathrm{Nn} 2]$ & 2.74 & $F 6 P[O 3 P]$ \\
\hline
\end{tabular}


Taking into account all of the above (sometimes contradictory) observations and hypotheses, it might be speculated that both anomers of F1,6BP may serve as a substrate for FBPase. However, the $\beta$-anomer of fructose-6-phosphate, because of its higher affinity for the enzyme, will be preferably observed in the FBPase crystal structures, even though under physiological conditions the $\alpha$-anomer of F1,6BP might be the true substrate of FBPase.

There is only one structure of mammalian FBPase in complex with fructose-1,6-bisphosphate deposited in the PDB. That structure of the porcine liver isozyme $(1 \mathrm{FBH})$ was modeled with the active site occupied by a superposition of the $\alpha$ - and $\beta$-anomers in 0.2:0.8 ratio. It is difficult to believe that a ligand molecule with only light atoms could be reliably modeled at 0.2 occupancy, especially at $2.5 \AA$ resolution. It is rather likely that the authors were trying to mimic the active site occupancy with the natural abundance of the $\alpha$ - and $\beta$-anomers known from solution. Unfortunately, it is not possible to validate this conclusion of the original authors (Zhang et al., 1993) in the electron density maps because no structure factor data were deposited together with the atomic coordinates. Moreover, the spatial arrangement of the two isomers in that model closely resembles the situations known from the structures containing the 2,5-anhydro analog of the substrate (Villeret et al., 1995). In contrast, in the structures presented in this work the $\beta$-anomer of the substrate was modeled in excellent and unambiguous electron density maps with the resolution as high as $1.92 \AA$. The anomeric configuration of the F1,6BP substrate is easy to determine with the help of the phosphate group at the C1 atom. Modeling is markedly more difficult in the case of F6P. There are 45 structures of mammalian FBPase in complex with the $\beta$-anomer of F6P in the PDB, and only one with the $\alpha$-anomer.

In summary, in this paper we have presented, for the first time, the crystal structure of human muscle FBPase in complex with its F1,6BP substrate, as well as its F6P product at full occupancy. Also, to the best of our knowledge, this is the first SAXS evidence for both, the $\mathrm{R}$ - and T-states of this enzyme in solution, so far known only from crystal structures.

\section{Author contribution}

JB: conducted structure refinements, analyzed the results, wrote the manuscript. KS: conducted the SAXS experiments and interpreted their results. JW: carried out protein expression and purification. RK: conducted most of the crystallographic experiments and calculations. DR: interpreted the biological context of the results, wrote the manuscript. MJ: coordinated the crystallographic part of the project, analyzed the results, wrote the manuscript. AD: conceived and coordinated the project, analyzed the results, wrote the manuscript.

\section{Acknowledgments}

We thank HZB for the allocation of synchrotron radiation beamtime. We are indebted to SAXS beamlines: I911-4 at MAX IV Laboratory in Lund, Sweden and P12 of the EMBL at the Petra-III storage ring.

\section{REFERENCES}

Afonine P, Grosse-Kunstleve R, Echols N, Headd J, Moriarty N, Mustyakimov M, Terwilliger T, Urzhumtsev A, Zwart P, Adams P (2012) Towards automated crystallographic structure refine- ment with phenix.refine. Acta Cryst D 68: 352-367. https://doi. org/10.1107/S0907444912001308

Al-Robaiy S, Eschrich K (1999) Rat muscle fructose-1,6-bisphosphatase: Cloning of the cDNA, expression of the recombinant enzyme, and expression analysis in different tissues. Biol Chem 380: 1079-1085. https://doi.org/10.1515/BC.1999.134

Barciszewski J, Wisniewski J, Kolodziejczyk R, Jaskolski M, Rakus D, Dzugaj A (2016) T-to-R switch of muscle fructose-1,6-bisphosphatase involves fundamental changes of secondary and quaternary structure. Acta Cryst D 72: 536-350. https://doi.org/10.1107/ S2059798316001765

Bartrons R, Hue L, Van Schaftingen E, Hers H (1983) Hormonal-control of fructose 2,6-bisphosphate concentration in isolated rat hepatocytes. Biochem I 214: 829-837. https://doi.org/10.1042/bj2140829

Benkovic S, deMaine M (1982) Mechanism of action of fructose 1,6-bisphosphatase. Adv Ensymol Relat Areas Mol Biol 53: 45-82

Choe J, Fromm H, Honzatko R (2000) Crystal structures of fructose 1,6-bisphosphatase: Mechanism of catalysis and allosteric inhibition revealed in product complexes. Biochemistry 39: 8565-8574. https:// doi.org/10.1021/bi000574g

Choe J, Poland B, Fromm H, Honzatko R (1998) Role of a dynamic loop in cation activation and allosteric regulation of recombinant porcine, fructose-1,6-bisphosphatase. Biochemistry 37: 11441-11450. https://doi.org/10.1021/bi981112u

Dzugaj A, Weber G, Weber C, Cocco L (2006) Localization and regulation of muscle fructose-1,6-bisphosphatase, the key enzyme of glyconeogenesis. Adv Enz Reg 46: 51-71. https://doi.org/10.1016/j. advenzreg.2006.01.021

Emsley P, Lohkamp B, Scott W, Cowtan K (2010) Features and development of Coot. Acta Cryst D 66: 486-501. https://doi. org/10.1107/S0907444910007493

Frey W, Fishbein R, Maine M, Benkovic S (1977) Substrate form of D-fructose 1,6-bisphosphate utilized by fructose 1,6-bisphosphatase. Biochemistry 16: 2479-2484. https://doi.org/10.1021/bi00630a025

Gao Y, Shen L, Honzatko R. (2014) Central cavity of fructose-1,6-bisphosphatase and the evolution of AMP/fructose 2,6-bisphosphate synergism in eukaryotic organisms. J Biol Chem 289: 8450-8461. https://doi.org/10.1074/jbc.M114.548586

Gidhjain M, Zhang Y, Vanpoelje P, Liang J, Huang S, Kim J, Elliott J, Erion M, Pilkis S, Elmaghrabi M, Lipscomb W (1994) The allosteric site of human liver fructose-1,6-bisphosphatase - analysis of 6 AMP site mutants based on the crystal-structure. J Biol Chem 269: $27732-27738$

Gizak A, Maciaszczyk E, Dzugaj A, Eschrich K, Rakus D (2008) Evolutionary conserved $\mathrm{N}$-terminal region of human muscle fructose 1,6-bisphosphatase regulates its activity and the interaction with aldolase. Prot Struc Func Bioinfo 72: 209-216. https://doi. org/10.1002/prot.21909

Gizak A, Maikowski M, Dus D, Dzugaj A (2004) Calcium inhibits muscle FBPase and affects its intracellular localization in cardiomyocytes. Febs Lett 576: 445-448. https://doi.org/10.1016/j.febslet.2004.09.050

Gizak A, Mazurek J, Wozniak M, Maciaszczyk-Dziubinska E, Rakus D (2013) Destabilization of fructose 1,6-bisphosphatase-Z-line interactions is a mechanism of glyconeogenesis down-regulation in vivo. Biochim Biophys Acta 1833: 622-628. https://doi.org/10.1016/j. bbamcr.2012.11.028

Gizak A, Pirog M, Rakus D (2012) Muscle FBPase binds to cardiomyocyte mitochondria under glycogen synthase kinase- 3 inhibition or elevation of cellular Ca2+ level. Febs Lett 586: 13-19. https:// doi.org/10.1016/j.febslet.2011.11.032

Gizak A, Wrobel E, Moraczewski J, Dzugaj A (2006) Changes in subcellular localization of fructose 1,6-bisphosphatase during differentiation of isolated muscle satellite cells. Febs Lett 580: 4042-4046. https://doi.org/10.1016/j.febslet.2006.06.042

Gizak A, Zarzycki M, Rakus D (2009) Nuclear targeting of FBPase in HL-1 cells is controlled by beta-1 adrenergic receptor-activated $\mathrm{G}(\mathrm{s})$ protein signaling cascade. Biocbim Biophys Acta 1793: 871-877. https:// doi.org/10.1016/j.bbamcr.2009.02.005

Kabsch W (2010) XDS. Acta Cryst D 66: 125-132. https://doi. org $/ 10.1107 /$ S0907444909047337

Kabsch W, Sander C (1983) Dictionary of protein secondary structure - Pattern-Recognition Of Hydrogen-Bonded And Geometrical Features. Biopolymers 22: 2577-2637. https://doi.org/10.1002/ bip.360221211

Ke H, Zhang Y, Liang J, Lipscomb W (1991) Crystal structure of the neutral form of fructose-1,6-bisphosphatase complexed with the product fructose 6-phosphate at 2.1-A resolution. Proc Natl Acad Sci USA 88: 2989-2993. https://doi.org/10.1073/pnas.88.8.2989

Konarev P, Volkov V, Sokolova A, Koch M, Svergun D (2003) PRIMUS: a Windows PC-based system for small-angle scattering data analysis. J Appl Crys 36: 1277-1282. https://doi.org/10.1107/ S0021889803012779

Krug M, Weiss M, Heinemann U, Mueller U (2012) XDSAPP: a graphical user interface for the convenient processing of diffraction 
data using XDS. J Appl Crys 45: 568-572. https://doi.org/10.1107/ S0021889812011715

Liu F, Fromm H (1988) Interaction of fructose 2,6-bisphosphate and AMP with fructose-1,6-bisphosphatase as studied by Nuclear Magnetic-Resonance spectroscopy. J Biol Chem 263: 9122-9128

Loffler T, Al-Robaiy S, Bigl M, Eschrich K, Schliebs R (2001) Expression of fructose-1,6-bisphosphatase mRNA isoforms in normal and basal forebrain cholinergic lesioned rat brain. Int J Dev Neurosci 19: 279-285. https://doi.org/10.1016/S0736-5748(01)00011-9

Mamczur P, Sok A, Rzechonek A, Rakus D (2012) Cell cycle-dependent expression and subcellular localization of fructose 1,6-bisphosphatase. Histochem Cell Biol 137: 121-136. https://doi.org/10.1007/ s00418-011-0884-1

Marcus F (1976) Interaction of salicylate at AMP site of fructose 1,6-bisphosphatase. Febs Lett 70: 159-162. https://doi.org/10.1016/00145793(76)80748-X

McCoy A, Grosse-Kunstleve R, Adams P, Winn M, Storoni L, Read R (2007) Phaser crystallographic software. J Appl Crys 40: 658-674. https://doi.org/10.1107/S0021889807021206

Nelson S, Choe J, Honzatko R, Fromm H (2000) Mutations in the hinge of a dynamic loop broadly influence functional properties of fructose-1,6-bisphosphatase. J Biol Chem 275: 29986-29992. https:// doi.org/10.1074/jbc.M000473200

Otwinowski Z, Minor W (1997) Processing of X-ray diffraction data collected in oscillation mode. Macromol Crystal 276: 307-326. https://doi.org/10.1016/S0076-6879(97)76066-X

Penhoet E, Kochman M, Rutter W (1969) Isolation of fructose diphosphate aldolases A, B, and C. Biochemistry 8: 4391-4395. https:// doi.org/10.1021/bi00839a025

Petoukhov M, Franke D, Shkumatov A, Tria G, Kikhney A, Gajda M, et al. (2012) New developments in the ATSAS program package for small-angle scattering data analysis. J Appl Crys 45: 342-350. https://doi.org/10.1107/S0021889812007662

Pilkis S, Claus T, Kurland I, Lange A (1995) 6-Phosphofructo-2-kinase/fructose-2,6-bisphosphatase - a metabolic signaling Ensyme Annu Rev Biochem 64: 799-835. https://doi.org/10.1146/annurev. bi.64.070195.004055

Pirog M, Gizak A, Rakus D (2014) Changes in quaternary structure of muscle fructose-1,6-bisphosphatase regulate affinity of the enzyme to mitochondria. Int J Biochem Cell Biol 48: 55-59. https://doi. org/10.1016/j.biocel.2013.12.015

Rakus D, Gizak A, Kasprzak A, Zarzycki M, Maciaszczyk-Dziubinska E, Dzugaj A (2013) The mechanism of calcium-induced inhibition of muscle fructose 1,6-bisphosphatase and destabilization of glyconeogenic complex. Plos One 8. https://doi.org/10.1371/journal. pone.0076669

Rakus D, Pasek M, Krotkiewski H, Dzugaj A (2004) Interaction between muscle aldolase and muscle fructose 1,6-bisphosphatase results in the substrate channeling. Biochemistry 43: 14948-14957. https://doi.org/10.1021/bi048886x

Rakus D, Tillmann H, Wysocki R, Ulaszewski S, Eschrich K, Dzugaj A (2003) Different sensitivities of mutants and chimeric forms of human muscle and liver fructose-1,6-bisphosphatases towards AMP. Biol Chem 384: 51-58. https://doi.org/10.1515/BC.2003.006

Rundqvist L, Aden J, Sparrman T, Wallgren M, Olsson U, Wolf-Watz M (2009) Noncooperative folding of subdomains in adenylate kinase. Biochemistry 48: 1911-1927. https://doi.org/10.1021/bi8018042

Schrank T, Bolen D, Hilser V (2009) Rational modulation of conformational fluctuations in adenylate kinase reveals a local unfolding mechanism for allostery and functional adaptation in proteins. Proc Natl Acad Sci USA 106: 16984-16989. https://doi.org/10.1073/ pnas.0906510106

Shi R, Chen Z, Zhu D, Li C, Shan Y, Xu G, et al. (2013) Crystal Structures of human muscle fructose-1, 6-bisphosphatase: novel quaternary states, enhanced AMP affinity, and allosteric signal transmission pathway. Plos One 8. https://doi.org/10.1371/journal. pone.0071242

Svergun D (1999) Restoring low resolution structure of biological macromolecules from solution scattering using simulated annealing. Biophys J 76: 2879-2886. https://doi.org/10.1016/S00063495(99) 777443-6

Svergun D, Barberato C, Koch M (1995) CRYSOL - A program to evaluate $\mathrm{x}$-ray solution scattering of biological macromolecules from atomic coordinates. J Appl Crys 28: 768-773. https://doi. org/10.1107/S0021889895007047

Tejwani G (1983) Regulation of fructose-bisphosphatase activity. Adv Ensymol Relat Areas Mol Biol 54: 121-194

Terwilliger T, Read R, Adams P, Brunger A, Afonine P, Grosse-Kunstleve R, Hung L (2012) Improved crystallographic models through iterated local density-guided model deformation and reciprocal-space refinement. Acta Cryst D 68: 861-870. https://doi.org/10.1107/ S0907444912015636

Van Schaftingen E, Hers H (1981) Inhibition of fructose-1,6-bisphosphatase by fructose 2,6-bisphosphate. Proc Natl Acad Sci USA 78: 2861-2863

Villeret V, Huang S, Fromm H, Lipscomb W (1995) Crystallographic evidence for the action of potassium, thallium, and lithium ions on fructose-1,6-bisphosphatase. Proc Natl Acad Sci USA 92: 8916-8920. https://doi.org/10.1073/pnas.92.19.8916

Villeret V, Huang S, Zhang Y, Lipscomb W (1995) Structural aspects of the allosteric inhibition of fructose-1,6-bisphosphatase by AMP - the binding of both the substrate-analog 2,5-anhydro-D-glucitol 1,6-bisphosphate and catalytic metal-ions monitored by X-ray crystallography. Biochemistry 34: 4307-4315. https://doi.org/10.1021/ bi00013a020

Winn M, Isupov M, Murshudov G (2001) Use of TLS parameters to model anisotropic displacements in macromolecular refinement. Acta Cryst D 57: 122-133. https://doi.org/10.1107/S0907444900014736

Winn M, Murshudov G, Papiz M, Carter C, Sweet R (2003) Macromolecular TLS refinement in REFMAC at moderate resolutions. Macromol Crystal 374: 300-321. https://doi.org/10.1016/S00766879(03)74014-2

Wisniewski J, Pirog M, Holubowicz R, Dobryszycki P, McCubrey J, Rakus D, Gizak, A (2017) Dimeric and tetrameric forms of muscle fructose-1,6-bisphosphatase play different roles in the cell. Oncotarget 8: 115420-115433. https://doi.org/10.18632/oncotarget.23271

Zarzycki M, Kolodziejczyk R, Maciaszczyk-Dziubinska E, Wysocki R, Jaskolski M, Dzugaj A (2011) Structure of E69Q mutant of human muscle fructose-1,6-bisphosphatase. Acta Cryst D 67: 1028-1034. https://doi.org/10.1107/S090744491104385X

Zarzycki M, Maciaszczyk E, Dzugaj A (2007) Glu 69 is essential for the high sensitivity of muscle fructose-1,6-bisphosphatase inhibition by calcium ions. Febs Lett 581: 1347-1350. https://doi. org/10.1016/j.febslet.2007.02.051

Zhang Y, Liang J, Huang S, Lipscomb W (1994) Toward a mechanism for the allosteric transition of pig-kidney fructose-1,6-bisphosphatase. J Mol Biol 244: 609-624. https://doi.org/10.1006/ jmbi.1994.1755 